\title{
Synthesis, antibacterial, and antioxidant studies of 7-amino-3-(4-fluorobenzoyl)indolizine-1-carboxylate derivatives
}

Vijayakumar Uppar ${ }^{1}$, Sandeep Chandrashekharappa ${ }^{3 *}$, Atiyaparveen I. Basarikatti ${ }^{1}$, Govindappa Banuprakash ${ }^{2}$, Mahendra K. Mohan $^{3}$, Mallikarjun Chougala ${ }^{4}$, Kiran K. Mudnakudu-Nagaraju ${ }^{5}$, Raghu Ningegowda ${ }^{6}$ and Basavaraj Padmashali ${ }^{1 *}$

${ }^{1}$ Department of Chemistry, School of Basic Sciences, Rani Channamma University, Belagavi-591156, India.

${ }^{2}$ Department of Chemistry, SJB Institute of Technology, Dr.Vishnuvardan road, Kengeri, Bengaluru-560060, India.

${ }^{3}$ Institute for Stem Cell Biology and Regenerative Medicine, NCBS, TIFR, GKVK-Campus Bellary Road, Bangalore-560065, India.

${ }^{4}$ Department of Biotechnology, JSS College of Arts, Commerce and Science Autonomous, Ooty Road, Mysore 570025, India.

${ }^{5}$ Division of Biotechnology \& Bioinformatics, Faculty of Life Sciences, JSS Academy of Higher Education \& Research, Mysuru-570015, India.

${ }^{6}$ Department of Chemistry, Jyoti Nivas College Autonomous, Bangalore-560095, Karnataka, India.

\begin{tabular}{l}
\hline ARTICLE INFO \\
\hline Received on: 08/10/2019 \\
Accepted on: 18/11/2019 \\
Available online: 05/02/2020 \\
\\
\hline Key words: \\
Indolizine, 4-aminopyridine, \\
acetylene, anti-bacterial, \\
antioxidant.
\end{tabular}

\begin{tabular}{l}
\hline ABSTRACT \\
In the present work, the quaternary salts of 4-aminopyridine, i.e., 4-amino-1-[2-(4-bromophenyl)-2-oxoethyl]pyridin-1-ium \\
bromides were obtained by stirring 4-aminopyridine with phenacyl bromides in acetone at room temperature separately. \\
These quaternary salts of 4-aminopyridine were treated with acetylenes (electron deficient), in the presence of anhydrous \\
possium carbonate in N,N-dimethylformamide solvent to get indolizine derivatives. The structures of newly synthesised \\
compounds have been confirmed by spectroscopic techniques, such as liquid chromatography mass spectrometry, \\
${ }^{1} \mathrm{H}-\mathrm{NMR}$, and elemental analysis. Synthesized all compounds were screened for antibacterial and antioxidant activity. \\
The compounds $2 \mathrm{e}, 2 \mathrm{~g}$, and $2 \mathrm{j}$ shows inhibition zone against bacteria and compounds $2 \mathrm{a}$ and $2 \mathrm{f}$ moderately active against \\
bacteria. All compounds $2 \mathrm{a}$ to $2 \mathrm{j}$ show 1,1 -diphenyl-2-picrylhydrazide radical free radical scavenging activity, Nitric \\
Oxide free radical scavenging activity, Reducing power scavenging activity, and Lipid peroxidation inhibition activity.
\end{tabular}

\section{INTRODUCTION}

The heterocyclic chemistry is one of the important class of organic chemistry because of their diversity of activity in nature and significant of synthesized compounds (Mallikarjun et al., 2016, Nagesh et al., 2014;2015; Rakshita et al., 2019; Siddesh et al., 2013; 2014a; 2014b; Thriveni et al., 2014). In this class, indolizine very interesting compound due to its wild range of biological activity, such as in vitro COX-2 inhibitory activity (Sandeep et al., 2018b), anti-tubercular activity (Swinborne et al., 2016), in vitro anticancer

\section{"Corresponding Author}

Basavaraj Padmashali, Department of Chemistry, School of Basic Sciences, Rani Channamma University, Belagavi 591156, India;

E-mail: basavarajpadmashali@yahoo.com; Tel.: +919844218894 Sandeep Chandrashekharappa, Institute for Stem Cell Biology and Regenerative Medicine, NCBS, TIFR, GKVK-Campus Bellary Road, Bangalore-560065, India.E-Mail: sandeep_m7@rediffmail.com; Tel.: +919448639413

activity (Sandeep et al., 2016a), antimicrobial activity (Sandeep et al., 2013a), larvicidal activity against Anopheles arabiensis (Sandeep et al., 2018a, 2016b). Indolizine is an organic molecule contaning both a p-excessive pyrrole and a p-deficient pyridine ring with only one bridged nitrigen. The indolizine system being isomeric with indole (Flitsch et al., 1984; Venugopal et al., 2019) and its synthesis also take our attention (Sandeep et al., 2013b; 2014; Kemnitzer et al., 2008). Compound 1 shows antibacterial and antifungal activity and compound 2 shows antibacterial activity (Hazra et al., 2011). In this present work, we have undertaken the synthesis of indolizine derivatives $2 \mathrm{a}-\mathrm{j}$. The final targeted moiety has been achieved in two steps and depicted in Scheme 1.

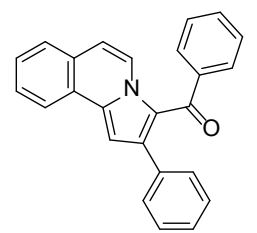

Compound 1

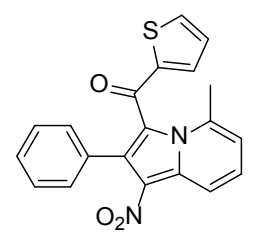

compound 2 


\section{MATERIALS AND METHODS}

\section{Chemistry}

All the chemicals and anhydrous solvents used in this work of analytical reagent grade and purchased from Sigma-Aldrich. Synthesized compounds IR spectra recorded on Bruker alpha FTIR spectrometer using Nujol. ${ }^{1} \mathrm{H}$ NMR spectra were recorded using $\mathrm{CDCl}_{3}$ and DMSO as a solvent, in Bruker AV 300. All chemical shifts are expressed in $\delta$ ppm. Using Jole JMS-D 300 mass spectrometer mass spectra were recorded at $70 \mathrm{eV}$. Elemental analysis was done on an Elementar Vario EL analyzer Satisfactory C, H, N analyses were obtained for all the compounds. The progress of reactions was monitored and checked by TLC, and further purification was finished by column chromatography using 60-120 mesh silica gel. All the melting points were recorded in open capillary and were uncorrected. Physiochemical constants like molecular mass, cLogP, melting point and $\%$ of the yield of Ethyl 7-amino-3-(4-bromobenzoyl)indolizine1 -carboxylate derivatives $(2 \mathrm{a}-2 \mathrm{j})$ provided in table 1 .

Preparation of 4-amino-1-(2-(4-bromophenyl)-2-oxethyl) pyridine-1-ium bromide (1d)

To a stirred of 4-Aminopyridine $(1.0 \mathrm{~g}, 0.0160 \mathrm{~mol})$ in anhydrous acetone $(10 \mathrm{ml})$ was added 2,4-Dibromoacetophenone $(2.93 \mathrm{~g}, 0.0106 \mathrm{~mol})$ and stirred for 30 minutes at normal room temperature. Solid was obtained which filtered and under vasuum to get quartenary salt 4-Amino -1-(2-(4-bromophenyl)-2-oxethyl) pyridine-1-ium bromide ( $89 \%$ yield).

\section{Preparation of ethyl-7-amino-3-(4-bromobenzyl)indolizine-1 -carboxylate (2g)}

To a stirred solution of 4-Amino-1-(2-(4-bromophenyl)2-oxoethyl)pyridine-1-ium bromide ( $0.5 \mathrm{~g}, 0.0013 \mathrm{~mol})$ in dry DMF was added ethylpropiolate $(0.1 \mathrm{~g}, 0.0013 \mathrm{~mol})$ [for compound $2 \mathrm{~h}$, dimethyl but-2-ynedioate $(0.1 \mathrm{~g}, 0.0013 \mathrm{~mol})], \mathrm{K}_{2} \mathrm{CO}_{3}(0.2 \mathrm{~g}, 0.0016$ $\mathrm{mol})$, and was stirred for 30 minutes in room temperature. The progrss of the reaction was monitored by TLC. After completion, the reaction mass was pour into container which contain crushed ice and filtered on the vacuum pump. The crude compound was purified by column chromatography using hexane:ehtlacetate as an eluent to afford ethyl-7-amino-3-(4-bromobenzoyl)indolizine1 -carboxylate $(78 \%$ yield). The remaining indolizine derivatives were synthesized by using the same protocol. The series of reaction carried out have been represented in Scheme 1.

Ethyl 7-amino-3-(4-fluorobenzoyl)indolizine-1-carboxylate (2a) ${ }^{1} \mathrm{H}-\mathrm{NMR}\left(600 \mathrm{MHz}, \mathrm{DMSO}-\mathrm{d}_{6}\right) ; \delta=9.53-9.52(1 \mathrm{H}, \mathrm{m})$, $7.77(1 \mathrm{H}, \mathrm{s}), 7.37$ (2H, s), 7.35-7.27 (4H, m), 6.69-6.64 (3H, m), $4.22-4.18(2 \mathrm{H}, q, J=7.2 \mathrm{~Hz}), 1.28-1.26(3 \mathrm{H}, t, J=7.2 \mathrm{~Hz})$; ${ }^{13} \mathrm{C}-\mathrm{NMR}\left(150 \mathrm{MHz}, \mathrm{DMSO}-\mathrm{d}_{6}\right) ; \delta=188.26,186.60,164.88$, $163.76,150.63,143.52,137.11,131.33,131.28,130.58,129.87$, $120.65,115.87,115.73,108.11,101.77,96.27,59.54$, and 14.94; MS (ESI, Positive): $\mathrm{m} / \mathrm{z}=327.2(\mathrm{M}+\mathrm{H})+$; analytical calculated for $\mathrm{C}_{18} \mathrm{H}_{15} \mathrm{FN}_{2} \mathrm{O}_{3} ; \mathrm{C}, 66.25, \mathrm{H}, 4.63,63, \mathrm{~N}, 8.58$; found, $\mathrm{C}, 66.19$, $\mathrm{H}, 4.59, \mathrm{~N}, 8.55$.

\section{Dimethyl 2-acetyl-7-amino-3-(4-fluorobenzoyl)indolizine-1,2 -dicarboxylate (2b)}

${ }^{1} \mathrm{H}-\mathrm{NMR}\left(600 \mathrm{MHz}, \mathrm{DMSO}-\mathrm{d}_{6}\right) ; \delta=9.60-9.62(1 \mathrm{H}, \mathrm{m})$, 7.78-7.72(2H,m), 7.70-7.68(2H,m), 7.41(1H,s), 7.00-7.10(3H,m), $3.89(3 \mathrm{H}, \mathrm{s}), 3.84(3 \mathrm{H}, \mathrm{s}) ;{ }^{13} \mathrm{C}-\mathrm{NMR}\left(150 \mathrm{MHz}, \mathrm{DMSO}-\mathrm{d}_{6}\right)$; $\delta=180.12,169.23,163.44,162.98,143.43,137.98,135.21$, $133.45,133.21,126.2,124.10,116.80,112.83,108.17,99.22$, 52.59, and 51.44; MS (ESI, Positive): $\mathrm{m} / \mathrm{z}=371.2(\mathrm{M}+\mathrm{H})^{+}$; analytical calculated for $\mathrm{C}_{19} \mathrm{H}_{15} \mathrm{FN}_{2} \mathrm{O}_{5} ; \mathrm{C}, 61.62, \mathrm{H}, 4.80, \mathrm{~N}, 7.56$; found $\mathrm{C}, 61.09, \mathrm{H}, 3.97, \mathrm{~N}, 7.18$.

Ethyl 7-amino-3-(2-nitrobenzoyl)indolizine-1-carboxylate (2c) 1H-NMR (600, DMSO-d $) ; \delta=9.48-9.47(1 \mathrm{H}, \mathrm{m}), 8.17$ $(1 \mathrm{H}, \mathrm{s}), 7.87-73(4 \mathrm{H}, \mathrm{m}), 7.27-7.25(1 \mathrm{H}, \mathrm{m}), 6.737 .70(3 \mathrm{H}, \mathrm{m})$, 4.19-4.15 (2H, $q, J=7.2 \mathrm{~Hz}), 1.23-1.20(3 \mathrm{H}, t, J=7.2 \mathrm{~Hz})$; ${ }^{13} \mathrm{C}-\mathrm{NMR}\left(150 \mathrm{MHz}, \mathrm{DMSO}-\mathrm{d}_{6}\right) ; \delta=178.83,163.52,151.05$, $147.60,143.74,135.76,134.42,131.37,130.54,130.15,129.49$, 125.13, 120.42, 110.10, 108.44, 59.64, and 14.54; MS (ESI, Positive): $\mathrm{m} / \mathrm{z}=354(\mathrm{M}+\mathrm{H})+$; analytical calculated for $\mathrm{C}_{18} \mathrm{H}_{15} \mathrm{~N}_{3} \mathrm{O}_{3}$; C, 61.19, H 4.28, N, 11.89; found C, 61.15, H, 4.22, N, 11.82 .

Dimethyl 7-amino-3-(2-nitrobenzoyl)indolizine-1,2 -dicarboxylate (2d)

1H-NMR (600 MHz, MNSO-d $), \delta=9.60-9.59(1 \mathrm{H}, \mathrm{m})$, $8.55(1 \mathrm{H}, \mathrm{s}), 8.43-8.25(2 \mathrm{H}, \mathrm{m}), 8.16-8.10(1 \mathrm{H}, \mathrm{m}), 7.86-7.77(1 \mathrm{H}$, m), 7.59-7.25 (1H, m), $6.80(2 \mathrm{H}, \mathrm{bs}), 3.87(3 \mathrm{H}, \mathrm{s}), 3.80(3 \mathrm{H}, \mathrm{s})$; ${ }^{13} \mathrm{C}-\mathrm{NMR}\left(150 \mathrm{MHz}, \mathrm{DMSO}-\mathrm{d}_{6}\right) ; \delta=165.22,163.05,151.43,146.14$,

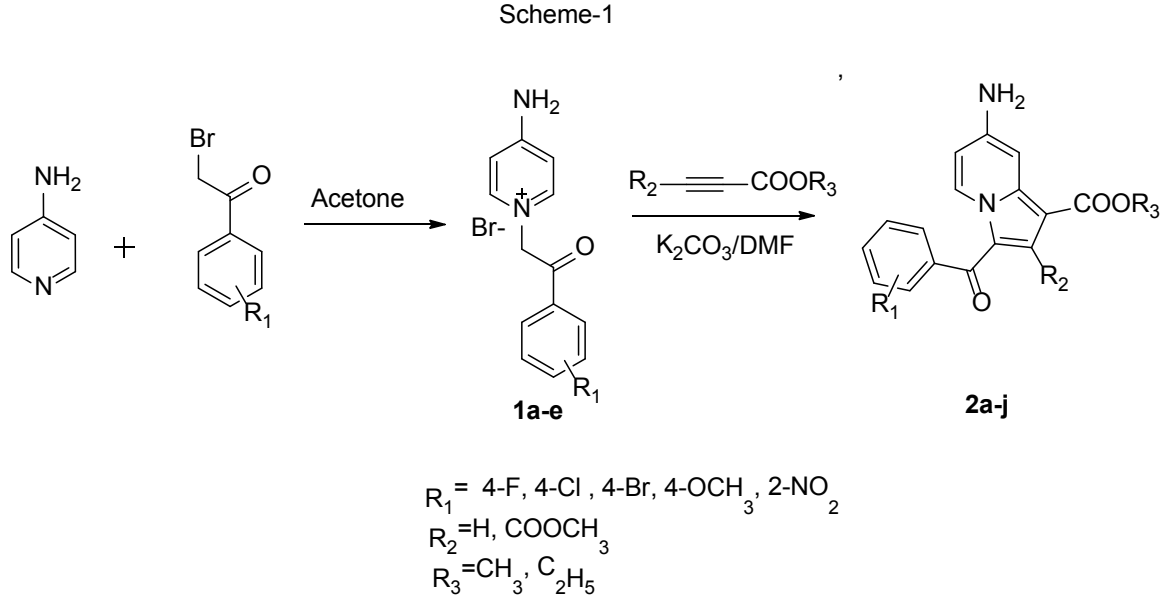


$141.89,135.17,134.79,134.18,133.73,132.71,131.47,130.73$, 130.64, 125.32, 124.66, 117.34, 109.27, 99.91, and 51.67; MS (ESI, Positive): $\mathrm{m} / \mathrm{z}=398(\mathrm{M}+\mathrm{H})^{+}$; analytical calcuted for $\mathrm{C}_{19} \mathrm{H}_{15} \mathrm{~N}_{3} \mathrm{O}_{7} ; \mathrm{C}$, 57.43, H, 3.81, N, 10.58, found; C, 57.40, H, 3.78, N, 10.52 .

\section{Ethyl 7-amino-3-(4-methoxybenzoyl)indolizine-1} -carboxylate (2e)

1H-NMR (600 MHz, DMSO-d $) ; \delta=9.52-9.51(1 \mathrm{H}, \mathrm{m})$, $7.78(1 \mathrm{H}, \mathrm{s}), 7.35(2 \mathrm{H}, \mathrm{s}), 7.25-7.19(4 \mathrm{H}, \mathrm{m}), 7.05-6.95$ $(3 \mathrm{H}, \mathrm{m}), 3.85(3 \mathrm{H}, \mathrm{s}), 4.22-4.18(2 \mathrm{H}, q, J=7.2 \mathrm{~Hz}), 1.28-1.26(3 \mathrm{H}, t$, $J=7.2 \mathrm{~Hz}) ;{ }^{13} \mathrm{C}-\mathrm{NMR}\left(150 \mathrm{MHz}, \mathrm{DMSO}-\mathrm{d}_{6}\right) ; \delta=188.26,186.60$, $164.88,163.76,150.63,143.52,137.11,131.33,131.28,130.58$, $129.87,120.65,115.87,115.73,108.11,101.77,96.27,59.54$, 55.86, and 14.94; MS (ESI Positive): $\mathrm{m} / \mathrm{z}=339.2(\mathrm{M}+\mathrm{H})+$; analytical calculated for $\mathrm{C}_{19} \mathrm{H}_{18} \mathrm{~N}_{2} \mathrm{O}_{4} ; \mathrm{C}, 6.44, \mathrm{H}, 5.36, \mathrm{~N}, 8.28$; found; C, 66.19, H, 5.19, N, 8.05.

Dimethyl 2-acetyl-7-amino-3-(4-methoxybenzoyl)indolizine -1,2-dicarboxylate (2f )

${ }^{1} \mathrm{H}-\mathrm{NMR}\left(600 \mathrm{MHz}, \mathrm{DMSO}-\mathrm{d}_{6}\right) ; \delta=9.67-9.65(1 \mathrm{H}, \mathrm{m})$, 7.88-7.82(2H, m), 7.80-7.78 (2H, m), $7.45(1 \mathrm{H}, \mathrm{s}), 7.10-7.12$ $(3 \mathrm{H}, \mathrm{m}), 3.90(3 \mathrm{H}, \mathrm{s}), 3.85(3 \mathrm{H}, \mathrm{s}), 3.83(3 \mathrm{H}, \mathrm{s}),{ }^{13} \mathrm{C}-\mathrm{NMR}(150$ MHz, DMSO-d $\left.{ }_{6}\right) ; \delta=181.23,166.22,165.27,164.98,142.55$, $138.23,135.26,131.25,129.66,126.24,124.87,114.66,112.83$, 108.67, 99.56, 56.35, 55.98, and 55.88; MS (ESI, Positive): $\mathrm{m} / \mathrm{z}$ $=383.2(\mathrm{M}+\mathrm{H})+$; analytical calculated for $\mathrm{C}_{20} \mathrm{H}_{18} \mathrm{~N}_{2} \mathrm{O}_{6} ; \mathrm{C}, 62.82$, H, 4.74, N, 7.33, found; C, 62.09, H, 4.57, N, 7.05.

\section{Ethyl 7-amino-3-(4-bromobenzoyl)indolizine-1-carboxylate (2g)}

${ }^{1} \mathrm{H}-\mathrm{NMR}\left(600 \mathrm{MHz}, \mathrm{DMSO}-\mathrm{d}_{6}\right) ; \delta=9.53-9.52(1 \mathrm{H}, \mathrm{m})$, 7.74-7.34 (2H, m), 7.65-7.63 (2H, m), $7.37(1 \mathrm{H}, \mathrm{s}), 7.27(1 \mathrm{H}, \mathrm{s})$, 6.96-6.67 (3H, m), 4.22-4.18 (2H, $q, J=7.2 \mathrm{~Hz}), .1 .28-1.24(3 \mathrm{H}, t$, $J=7.2 \mathrm{~Hz}) ;{ }^{13} \mathrm{C}-\mathrm{NMR}(150 \mathrm{MHz}$, DMSO-d $) ; \delta=184.56,163.71$, $150.75,143.59,139.67,131.85,130.80,130.63,130.00,124.91$, 120.55, 108.15, 102.00, 96.33, 59.57, and 14.93; MS (ESI, Positive): $\mathrm{m} / \mathrm{z}=387(\mathrm{M}+\mathrm{H})+$; analytical calculated for $\mathrm{C}_{18} \mathrm{H}_{15} \mathrm{~N}_{2} \mathrm{O}_{3}$; C, 55.83, H, 3.90, N, 7.23, found, C, 55.80, H, 3.87, N, 7.18.

\section{Dimethyl 2-acetyl-7-amino-3-(4-bromobenzoyl)indolizine-1,2} -dicarboxylate (2h)

${ }^{1} \mathrm{H}-\mathrm{NMR} \quad(600 \mathrm{MHz}, \quad$ DMSO-d $) ; \delta=9.59-9.58$ $(1 \mathrm{H}, \mathrm{m}), 7.75-7.73(2 \mathrm{H}, \mathrm{m}), .7 .70-7.68(2 \mathrm{H}, \mathrm{m}), 7.44(1 \mathrm{H}, \mathrm{s})$, 7.11-7.07 (3H, m), $3.92(3 \mathrm{H}, \mathrm{s}), 3.86(3 \mathrm{H}, \mathrm{s}) ;{ }^{13} \mathrm{C}-\mathrm{NMR}-(150$
MHz, DMSO-d $) \delta=178.12,168.43,163.11,142.56,136.67$, $134.33,133.17,132.89,125.12,124.00,116.47,112.49,108.01$, 99.78, 52.31, and 51.69; MS (ESI, Positive): $\mathrm{m} / \mathrm{z}=331.2(\mathrm{M}+\mathrm{H})+$; analytical calculate for $\mathrm{C}_{19} \mathrm{H}_{15} \mathrm{BrN}_{2} \mathrm{O}_{5} ; \mathrm{C}, 52.92, \mathrm{H}, 3.51, \mathrm{~N}, 6.50$; found; C, 52.09, H, 3.37, N, 6.08.

Ethyl 7-amino-3-(4-chlorobenzoyl)indolizine-1-carboxylate (2i)

1H-NMR (600 MHz, DMSO-d6); $\delta=9.52-9.51(1 \mathrm{H}$, m), 7.71-7.69 (2H, m), 7.60-7.59 (2H, m), 7.46-7.44(1H, m), 7.27-7.37 $(1 \mathrm{H}, \mathrm{m}), 6.69-6.66(3 \mathrm{H}, \mathrm{m}), 4.22-4.18(2 \mathrm{H}, q$ $J=7.2 \mathrm{~Hz}), 1.22-1.19(3 \mathrm{H}, t, J=7.2 \mathrm{~Hz}) ;{ }^{13} \mathrm{C}-\mathrm{NMR}(150 \mathrm{MHz}$, DMSO-d $) ; \delta=181.29,163.72,150.74,143.58,139.32,136.06$, $130.61,129.99,128.93,128.33,120.59,108.15,101.97,96.32$, 59.57, and 14.93; MS (ESI, Positive): $\mathrm{m} / \mathrm{z}=343(\mathrm{M}+\mathrm{H})+$, analytical calculated for $\mathrm{C}_{18} \mathrm{H}_{15} \mathrm{ClN}_{2} \mathrm{O}_{3} ; \mathrm{C}, 63.07, \mathrm{H}, 4.41, \mathrm{~N}, 8.17$; found; C, 62.89, H, 4.38, N, 8.14.

Dimethyl 2-acetyl-7-amino-3-(4-chlorobenzoyl)indolizine-1,2 -dicarboxylate $(2 \mathrm{j})$

${ }^{1} \mathrm{H}-\mathrm{NMR}\left(600 \mathrm{MHz}, \mathrm{DMSO}-\mathrm{d}_{6}\right) ; \delta=9.59-9.58(1 \mathrm{H}, \mathrm{m})$, 7.76-7.74 (2H, m), 7.71-7.69 (2H, m), $7.43(1 \mathrm{H}, \mathrm{s}), 7.10-7.06(3 \mathrm{H}$, m), $3.90(3 \mathrm{H}, \mathrm{s}), 3.85(3 \mathrm{H}, \mathrm{s}),{ }^{13} \mathrm{C}-\mathrm{NMR}\left(150 \mathrm{MHz}, \mathrm{DMSO}-\mathrm{d}_{6}\right)$ $\delta=179.12,169.43,163.02,142.43,136.98,134.21,133.93$, 133.41, 126.12, 124.10, 116.67, 112.79, 108.23, 99.01, 52.30, and 51.66; MS (ESI, Positive): $\mathrm{m} / \mathrm{z}=387.2(\mathrm{M}+\mathrm{H})+$, analytical calculated for $\mathrm{C}_{19} \mathrm{H}_{15} \mathrm{ClN}_{2} \mathrm{O}_{5} ; \mathrm{C}, 59.00, \mathrm{H}, 3.91, \mathrm{~N}, 7.24$; found; $\mathrm{C}$, $58.09, \mathrm{H}, 3.77, \mathrm{~N}, 7.08$

\section{Pharmacology studies}

\section{Antibactrerial activity}

The clean cultures obtained were revived and maintained in nutrient agar at $37^{\circ} \mathrm{C}$. The microorganisms were cultured in nutrient broth $37^{\circ} \mathrm{C}$ overnight.

\section{Determination of antibacterial activity by disk-diffusion method}

The test organisms were subculture using nutrient agar medium. The tubes containg sterilized medium were inoculated the respective bacterial strain. After incubation at $37 \pm 1{ }^{\circ} \mathrm{C}$ for 18 hours, they stored in a refrigerator. The nutrient agar medium was sterilized by autocleaving at $121^{\circ}$ for 15 minutes. In to each sterilized petri plate, was poured with LB Agar

Table 1. Physiochemical constants of Ethyl 7-amino-3-(4-bromobenzyol)indolizine-1-carboxylate derivatives (2a -2j).

\begin{tabular}{|c|c|c|c|c|c|c|c|}
\hline Compound no & Molicular formula (Mol mass) & $\mathbf{R}_{1}$ & $\mathbf{R}_{2}$ & $\mathbf{R}_{3}$ & Yield (\%) & $\operatorname{clog} P$ & $\mathrm{MP}$ in ${ }^{\circ} \mathrm{C}$ \\
\hline $2 \mathrm{a}$ & $\mathrm{C}_{18} \mathrm{H}_{15} \mathrm{FN}_{2} \mathrm{O}_{3}(326.11)$ & $4-\mathrm{F}$ & $\mathrm{H}$ & $\mathrm{C}_{2} \mathrm{H}_{5}$ & 72 & 3.66 & $170-172$ \\
\hline $2 b$ & $\mathrm{C}_{19} \mathrm{H}_{15} \mathrm{FN}_{2} \mathrm{O}_{3}(370.10)$ & $4-\mathrm{F}$ & $\mathrm{COOCH}_{3}$ & $\mathrm{CH}_{3}$ & 76 & 2.10 & $168-170$ \\
\hline $2 \mathrm{c}$ & $\mathrm{C}_{18} \mathrm{H}_{15} \mathrm{~N}_{3} \mathrm{O}_{5}(353.10)$ & $2-\mathrm{NO}_{2}$ & $\mathrm{H}$ & $\mathrm{C}_{2} \mathrm{H}_{5}$ & 74 & 3.18 & $183-185$ \\
\hline $2 d$ & $\mathrm{C}_{19} \mathrm{H}_{15} \mathrm{~N}_{3} \mathrm{O}_{5}(397.09)$ & $2-\mathrm{NO}_{2}$ & $\mathrm{COOCH}_{3}$ & $\mathrm{CH}_{3}$ & 84 & 1.62 & $174-176$ \\
\hline $2 \mathrm{e}$ & $\mathrm{C}_{19} \mathrm{H}_{18} \mathrm{~N}_{2} \mathrm{O}_{3}(338.13)$ & 4-OMe & $\mathrm{H}$ & $\mathrm{C}_{2} \mathrm{H}_{5}$ & 80 & 3.49 & $191-193$ \\
\hline $2 f$ & $\mathrm{C}_{20} \mathrm{H}_{18} \mathrm{~N}_{2} \mathrm{O}_{6}(382.12)$ & 4-OMe & $\mathrm{COOCH}_{3}$ & $\mathrm{CH}_{3}$ & 78 & 1.95 & $177-179$ \\
\hline $2 \mathrm{~g}$ & $\mathrm{C}_{18} \mathrm{H}_{15} \mathrm{BrN}_{2} \mathrm{O}_{3}(430.02)$ & $4-\mathrm{Br}$ & $\mathrm{H}$ & $\mathrm{C}_{2} \mathrm{H}_{5}$ & 75 & 3.42 & $169-171$ \\
\hline $2 \mathrm{~h}$ & $\mathrm{C}_{19} \mathrm{H}_{15} \mathrm{BrN}_{2} \mathrm{O}_{3}(386.03)$ & $4-\mathrm{Br}$ & $\mathrm{COOCH}_{3}$ & $\mathrm{CH}_{3}$ & 78 & 2.82 & $173-175$ \\
\hline $2 \mathrm{i}$ & $\mathrm{C}_{18} \mathrm{H}_{15} \mathrm{ClN}_{2} \mathrm{O}_{3}(386.07)$ & $4-\mathrm{Cl}$ & $\mathrm{H}$ & $\mathrm{C}_{2} \mathrm{H}_{5}$ & 81 & 4.23 & $163-165$ \\
\hline $2 \mathrm{j}$ & $\mathrm{C}_{19} \mathrm{H}_{15} \mathrm{ClN}_{2} \mathrm{O}_{3}(342.08)$ & $4-\mathrm{Cl}$ & $\mathrm{COOCH}_{3}$ & $\mathrm{CH}_{3}$ & 82 & 2.67 & $154-156$ \\
\hline
\end{tabular}

cLogP was calculated using ChemDraw Professional $16.0 \mathrm{v}$. 
medium. The Plates were left at room temperature aseptically to allow the solidification. After solidification, the appropriate bacterial cultures were inoculated over the surface of the agar using a sterile cotton swabbing method. Discs measuring $6 \mathrm{~mm}$ in diameter were used. The discs of the each compounds was placed individually on the LB agar mediumwith fresh bacteria respectively.

The antibacterial result of the all synthesized 10 ethyl 7-amino-3-(4-bromobenzoyl)indolizine-1-carboxylate compounds is summarized in Table 2. Among 10 compounds, only four compounds exhibited inhibition to bacteria and the remaining six compounds not active against tested bacteria.

\section{In vitro antioxidant studies}

\section{DPPH free radical inhibition assy}

The stable 1,1-diphenyl-2-picrylhydrazide radical (DPPH) was used for determining free radical scavenging activity. Different concentations compounds were added at an equal volume to the silution of DPPH $(500 \mu \mathrm{l})$. After the sample preparation, keep the sample for 20 minutes and the absorbance was recorded at $517 \mathrm{~nm}$ at room temperature, using Ultraviolet (UV) spectrophotometer. The experiment was repeated thrice. Ascorbic acid was used as standard control (Koleva et al., 2002).
The DPPH free radical scavenging activity was calculated using the fallowing formula:

$$
\% \text { Scavenging effect }=\frac{(\text { Control absorbtion }- \text { Test absorbance })}{\text { Control absorbtion }} \times 100
$$

All synthesized ethyl 7-amino-3-(4-bromobenzoyl) indolizine-1-carboxylate derivatives screened for their inhibition activity against DPPH free radical. The DPPH free radical scavenging result of the all synthesized ten ethyl 7-amino3 -(4-bromobenzoyl)indolizine-1-carboxylate compounds is summarized in Table 3 and graph of Table 3 is shown in Figure 1. All compounds moderately active against DPPH free radical with compare to standard ascorbic acid.

\section{Nitric oxide radical scavenging activity}

Nitric oxide was produced from sodium nitroprusside and measured by the Griess reagent. Nitric oxide spontaneously generated by sodium nitroprusside in aqueous solution at physiological $\mathrm{pH}$, this relised nitric oxide react with oxygen to produce nitrite ions that can estimated by the Griess reagent. Scavengers of nitric play race with oxygen leading to reduce the production of nitric oxide. $5 \mathrm{mM}$ of sodium nitropprusside in phosphate buffered saline was mixed with different aliquots of

Table 2. Antibacterial activity of Indolizines derivatives in agar-disc diffusion method.

\begin{tabular}{|c|c|c|c|c|c|c|}
\hline Compound & Bacillus subtilis & Escherichia coli & Pseudomonas aerudinous & Staphylococcus.aureus & Bacillus cereus & Zymomonas mobilis \\
\hline $2 a$ & $14 \pm 0.81$ & 00 & $10.5 \pm 0.5$ & 00 & $10.05 \pm 0.5$ & $13.0 \pm 1.0$ \\
\hline $2 \mathrm{~b}$ & 00 & 00 & 00 & 00 & 00 & 00 \\
\hline $2 \mathrm{c}$ & 00 & 00 & 00 & 00 & 00 & 00 \\
\hline $2 \mathrm{~d}$ & 00 & 00 & 00 & 00 & 00 & 00 \\
\hline $2 \mathrm{e}$ & $16.66 \pm 1.15$ & $14.66 \pm 1.15$ & $15.33 \pm 1.15$ & $14.33 \pm 2.08$ & $8.66 \pm 1.15$ & $15.66 \pm 0.57$ \\
\hline $2 \mathrm{f}$ & $10.0 \pm 0.81$ & 00 & 00 & 00 & 00 & 00 \\
\hline $2 \mathrm{~g}$ & $12.66 \pm 1.15$ & $15.66 \pm 1.15$ & $9.33 \pm 1.15$ & $8.66 \pm 1.15$ & $10.33 \pm 0.57$ & $8.66 \pm 1.15$ \\
\hline $2 \mathrm{~h}$ & 00 & 00 & 00 & 00 & 00 & 0 \\
\hline $2 \mathrm{i}$ & 00 & 00 & 00 & 00 & 00 & 00 \\
\hline $2 \mathrm{j}$ & $11.33 \pm 1.15$ & $15.33 \pm 0.57$ & $11.33 \pm 1.15$ & $11.66 \pm 0.57$ & $10.33 \pm 0.57$ & $11.33 \pm 1.15$ \\
\hline
\end{tabular}

(Diameter of the inhibition zone in $\mathrm{mm}$ at $100 \mu \mathrm{g}$ of Sample), $N=3 \pm \mathrm{SD}$.

Table 3. DPPH free radical scavenging activity of synthesized dimethyl 7-amino-3-benzoylindolizine-1,2-dicarboxylate derivatives.

\begin{tabular}{|c|c|c|c|c|c|}
\hline \multirow{2}{*}{$\frac{\text { Compd. number }}{\text { Concentration }(\mu \mathrm{g} / \mathrm{ml})}$} & \multicolumn{5}{|c|}{ Inhibition in \% } \\
\hline & 20 & 40 & 60 & 80 & 100 \\
\hline $2 a$ & 6.952194 & 20.0284 & 31.08308 & 33.67005 & 35.46838 \\
\hline $2 b$ & 8.874152 & 11.58933 & 17.78623 & 19.02114 & 27.69117 \\
\hline $2 \mathrm{c}$ & 3.573405 & 8.906978 & 13.81798 & 14.62787 & 18.83489 \\
\hline $2 \mathrm{~d}$ & 3.274101 & 13.22477 & 15.64046 & 17.19529 & 20.06601 \\
\hline $2 \mathrm{e}$ & 10.49616 & 30.28744 & 36.46627 & 38.52124 & 43.15716 \\
\hline $2 \mathrm{f}$ & 7.520194 & 12.2411 & 15.22927 & 18.33619 & 21.78378 \\
\hline $2 \mathrm{~g}$ & 4.53826 & 16.44915 & 26.76646 & 30.91653 & 32.83957 \\
\hline $2 \mathrm{~h}$ & 1.949414 & 3.367248 & 9.447937 & 11.71589 & 17.61575 \\
\hline $2 \mathrm{i}$ & 7.599865 & 22.64933 & 28.71759 & 34.22499 & 40.38501 \\
\hline $2 \mathrm{j}$ & 8.4359 & 15.60315 & 19.60633 & 27.39953 & 37.24206 \\
\hline Ascorbic acid & 27.57077 & 45.46394 & 63.46551 & 78.89519 & 86.31934 \\
\hline
\end{tabular}


2-10 $\mu \mathrm{g}$ the compound and incubated at temperature $29^{\circ} \mathrm{C}$ for 2 hours. The compound reacted with Griess reagent. The absorbtion of the chromophore produced during the diazotiazation of nitrite with sulphanilamide and subsequent coupling with naphthyl ethylene diamine was read at $550 \mathrm{~nm}$ (Marcocci et al., 1994). The nitric oxide free radical scavenging activity was calculated using thr fallowing formula:

$$
\% \text { Inhibition }=\frac{(\text { Absorbance control }- \text { Absorbance of sample })}{\text { Absorbance control }} \times 100
$$

All synthesized ethyl 7-amino-3-(4-bromobenzoyl) indolizine-1-carboxylate derivatives screened for their inhibition activity against nitric oxide free radical. The nitric oxide free radical scavenging result of the all synthesized 10 ethyl 7-amino- 3-(4-bromobenzoyl)indolizine-1-carboxylate compounds is summarised in Table 4 and graph of Table 4 is shown in Figure 2. All compounds moderately active against nitric oxide free radical with compare to standard ascorbic acid.

\section{Reducing power assay}

Different concentration of compound was mixed with an equal volume of $(2.5 \mathrm{ml})$ of $0.2 \mathrm{~mol}$ phosphate buffer $(6.6 \mathrm{pH})$ and $1 \%$ potassium ferricyanide $(2.5 \mathrm{ml})$ and incubated at $50^{\circ} \mathrm{C}$ for 20 minutes. 10\% Trichloroacetic acid $(2.5 \mathrm{ml})$ was added and centrifuged at 3,000 rpm for 10 minutes. The upper layer of the solution $(2.5 \mathrm{ml})$ was mixed with $2.5 \mathrm{ml}$ of water and $0.1 \%$ ferric chloride of $0.5 \mathrm{ml}$ and absorbance was read at $700 \mathrm{~nm}$. An increase in the absorbance of indicates increases in redusing power (Oyaizu et al., 1986).

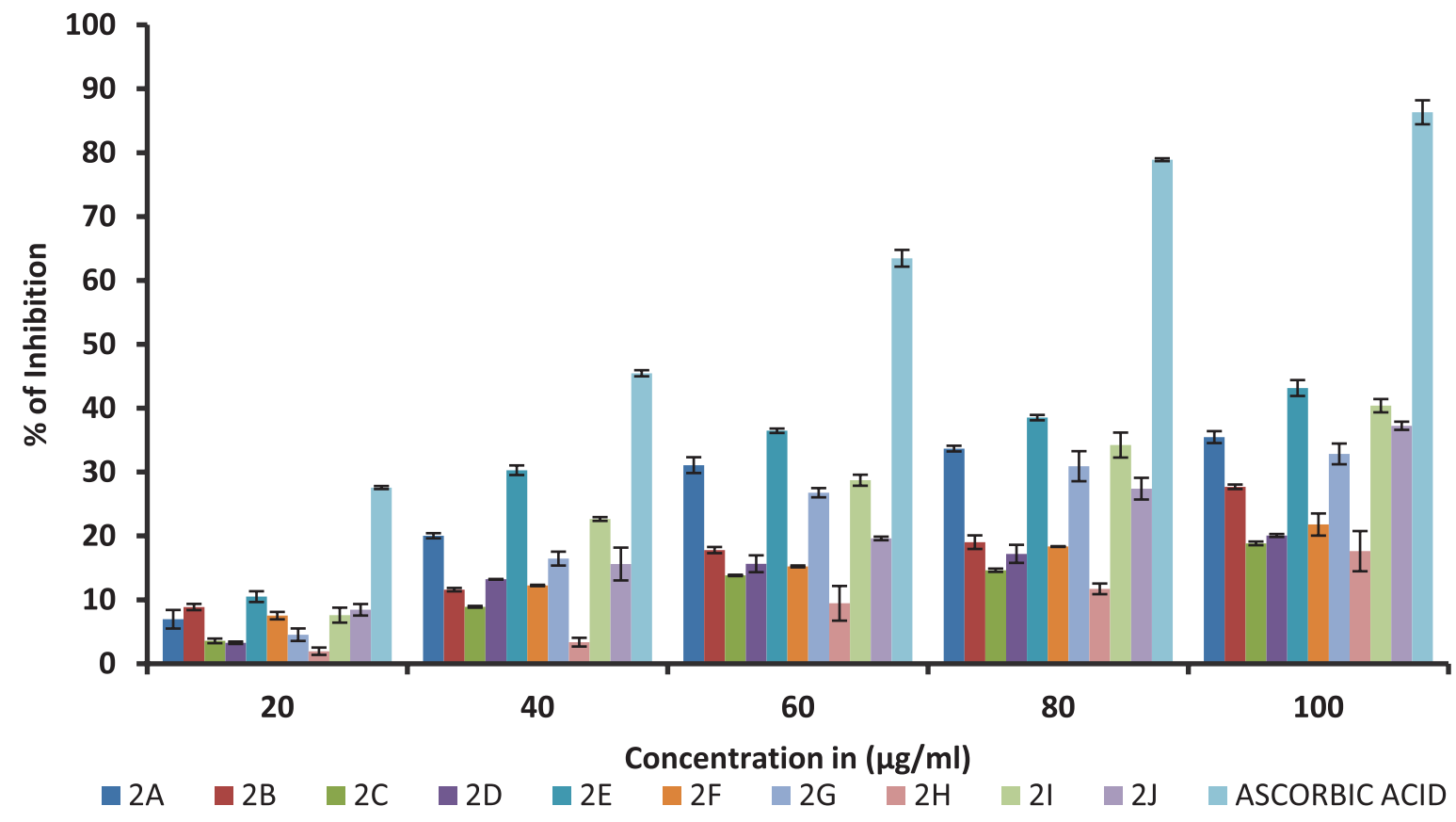

Figure 1. $\%$ of inhibition in free radical scavenging activity of dimethyl 7-amino-3-benzoylindolizine-1,2-dicarboxylate derivatives against $\mathrm{DPPH}$ free radical values were means $\pm \mathrm{SD}$ of triplicate.

Table 4. Nitric oxide free radical scavenging acticity of synthesized dimethyl 7-amino-3-benzoylindolizine-1,2-dicarboxylate derivatives.

\begin{tabular}{|c|c|c|c|c|c|}
\hline \multirow{2}{*}{$\frac{\text { Compd. number }}{\text { Concentration }(\mu \mathrm{g} / \mathrm{ml})}$} & \multicolumn{5}{|c|}{ Inhibition in \% } \\
\hline & 20 & 40 & 60 & 80 & 100 \\
\hline $2 \mathrm{a}$ & 8.609501 & 11.28838 & 16.79519 & 21.30167 & 24.43246 \\
\hline $2 b$ & 5.139437 & 8.355743 & 11.43443 & 16.06493 & 20.51322 \\
\hline $2 \mathrm{c}$ & 4.269065 & 13.22231 & 16.21296 & 18.22201 & 24.45212 \\
\hline $2 d$ & 2.574787 & 3.45503 & 5.907833 & 7.573848 & 13.37522 \\
\hline $2 \mathrm{e}$ & 1.251929 & 5.826586 & 17.28959 & 17.15912 & 19.485 \\
\hline $2 \mathrm{f}$ & 1.549592 & 10.90729 & 11.75832 & 16.52459 & 18.80608 \\
\hline $2 \mathrm{~g}$ & 4.72745 & 6.220635 & 9.695841 & 14.69706 & 17.38694 \\
\hline $2 \mathrm{~h}$ & 4.683 & 7.423 & 8.369 & 12.204 & 16.153 \\
\hline $2 \mathrm{i}$ & 5.097399 & 11.43091 & 13.20099 & 14.02766 & 17.57459 \\
\hline $2 \mathrm{j}$ & 2.199841 & 4.47381 & 7.80029 & 9.311806 & 11.5696 \\
\hline Ascorbic acid & 28.80784 & 42.53922 & 58.9574 & 72.23137 & 81.67745 \\
\hline
\end{tabular}


All synthesised ethyl 7-amino-3-(4-bromobenzoyl) indolizine-1-carboxylate derivatives screened for their reducing power. The potassium ferricyanide reduction power of the all synthesized 10 ethyl 7-amino-3-(4-bromobenzoyl)indolizine-1carboxylate compounds is summarized in Table 5 and graph of Table 5 is shown in Figure 3. All compounds moderately act as a reducing agents against ferric ion.

\section{Lipid peroxidation inhibition assay}

A reformed thiobarbituric acid-reactive species assy was used to measure the lipid peroxide formed. For this assay, egg yolk homogenates as lipid rich media. MDA (Malondialdihyde), a secondary end product of the oxidation of poly unsaturated fatty acids reacts with two molecules of TBA (thiobarbituric acid) yielding a pinkish red chromogen with an absorbance maximum at $532 \mathrm{~nm}$. Egg homogenate $0.5 \mathrm{ml}(10 \%$ in distilled water, v/v) and synthesized compound were mixed in a test tube and volume was made up to $1 \mathrm{ml}$ by adding distilled water. Finally, $0.05 \mathrm{ml}$ iron(II) sulfate $(0.07 \mathrm{M})$ was added to the above mixture and incubated for 30 minutes to induce lipid peroxidation. Thereafter, $1.5 \mathrm{ml}$ of $20 \%$ acetic acid ( $\mathrm{pH}$ adujest to 3.5 with sodium hydroxide) and $1.5 \mathrm{ml}$ of $0.8 \%$ thiobarbituric acid (w/v) (prepared in $1.1 \%$ sodim lauryl sulphate) and $0.05 \mathrm{ml} \mathrm{20 \%} \mathrm{TCA} \mathrm{was} \mathrm{added,} \mathrm{vortexes} \mathrm{and}$ then heted for 1 hour in a boling water bath. $5.0 \mathrm{ml}$ of butan-1-ol was added to each tube in cool condition and centrifuged at 3,000 rpm for 10 minutes. The absorpbance of organic upper layer was measured at $532 \mathrm{~nm}$ using UV spectrophotometer (Ruberto et al., 2000). The lipid peroxidation inhibition activity was calculated usingthe fallowing formula.

$\%$ Inhibition $=\frac{(\text { Absorbance of control }- \text { Absorbance of sample })}{\text { Absorbance of control }} \times 100$

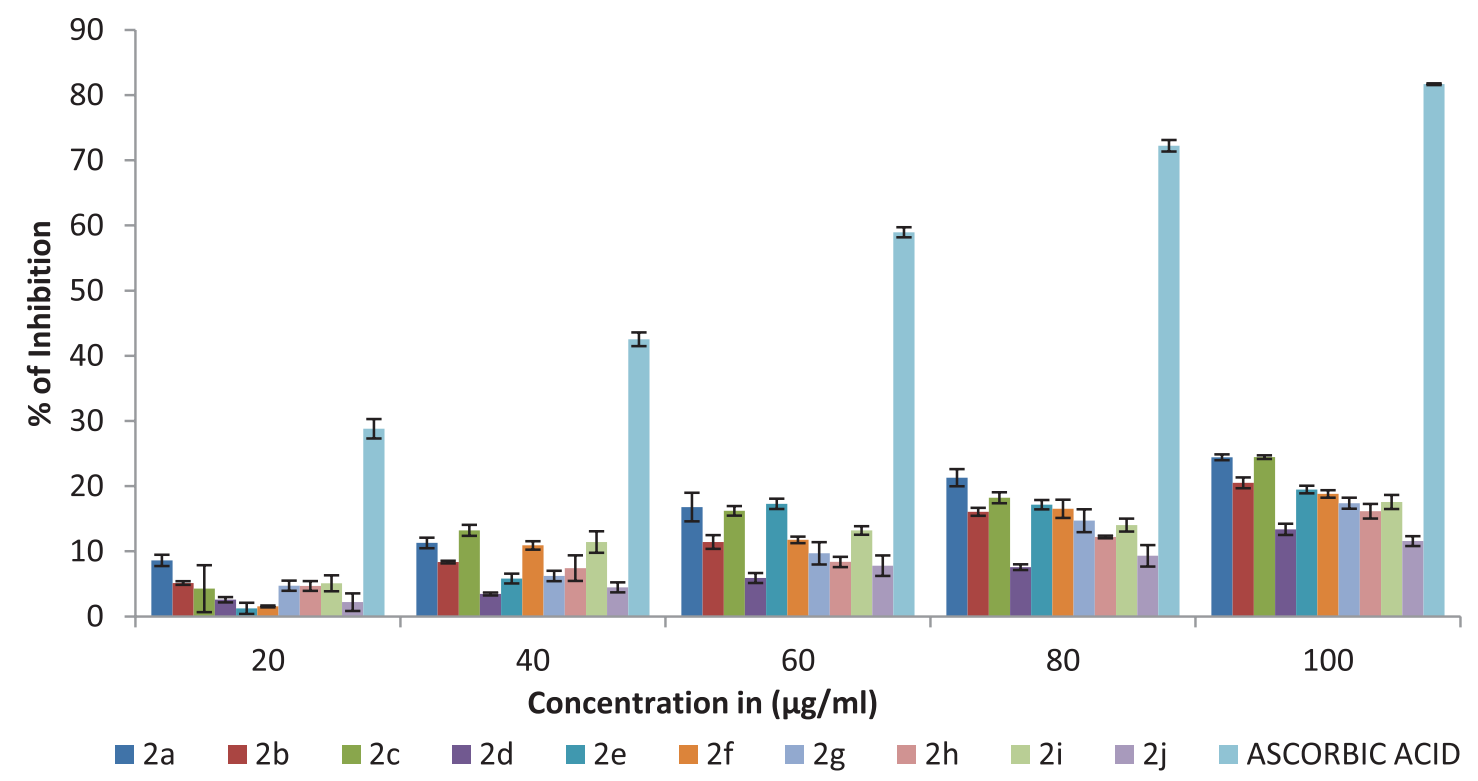

Figure 2. \% of inhibition in free radical scavenging activity of dimethyl 7-amino-3-benzoylindolizine-1,2-dicarboxylate derivatives against Nitric oxide free radical. Values were means \pm SD of triplicate.

Table 5. Reducing power scavenging activity of synthesized dimethyl 7-amino-3-benzoylindolizine-1,2-dicarboxylate derivatives.

\begin{tabular}{ccccccc}
\hline Compd. number & \multicolumn{7}{c}{ Inhibition in \% } \\
\hline Concentration $(\boldsymbol{\mu g} / \mathbf{m l})$ & $\mathbf{0}$ & $\mathbf{2 0}$ & $\mathbf{4 0}$ & $\mathbf{6 0}$ & $\mathbf{8 0}$ & $\mathbf{1 0 0}$ \\
\hline $2 \mathrm{a}$ & 0.109567 & 0.199967 & 0.2582 & 0.265533 & 0.3464 & 0.3797 \\
$2 \mathrm{~b}$ & 0.0964 & 0.124033 & 0.1107 & 0.141267 & 0.129033 & 0.139433 \\
$2 \mathrm{c}$ & 0.0964 & 0.127167 & 0.171133 & 0.288267 & 0.288767 & 0.352767 \\
$2 \mathrm{~d}$ & 0.105733 & 0.124233 & 0.140167 & 0.1501 & 0.209767 & 0.247933 \\
$2 \mathrm{e}$ & 0.1043 & 0.159633 & 0.316333 & 0.353633 & 0.396633 & 0.422033 \\
$2 \mathrm{f}$ & 0.131667 & 0.212667 & 0.296733 & 0.323433 & 0.300767 & 0.419467 \\
$2 \mathrm{~g}$ & 0.106633 & 0.141333 & 0.144067 & 0.180667 & 0.1536 & 0.168367 \\
$2 \mathrm{~h}$ & 0.147933 & 0.1742 & 0.208567 & 0.259633 & 0.326967 & 0.313667 \\
$2 \mathrm{i}$ & 0.088033 & 0.1182 & 0.1689 & 0.2211 & 0.253333 & 0.2984 \\
$2 \mathrm{j}$ & 0.086233 & 0.089667 & 0.0984 & 0.0927 & 0.099767 & 0.1063 \\
Ascorbic acid & 0.145167 & 0.42767 & 0.688367 & 0.965 & 1.2361 & 1.329133 \\
\hline
\end{tabular}


All synthesized ethyl 7-amino-3-(4-bromobenzoyl) indolizine-1-carboxylate derivatives screened for their lipid peroxidation inhibition activity. The lipid peroxidation inhibition of the all synthesized 10 ethyl 7-amino-3(4-bromobenzoyl)indolizine-1-carboxylate compounds is summarized in Table 6 and graph of Table 6 is shown in Figure 4. All compounds moderately act as reducing agents against lipid peroxidation.

\section{RESULTS AND DISCUSSION}

\section{Statistical analysis}

The resultrs of the experiment was expressed as mean \pm SD of triplicate. All stastical analysis and graphing of data were accomplished using MS-excel software

\section{Chemistry}

In this present work, the novel indolizine derivatives were synthesized with eco-friendly and reduced temperature method. The time required to complete the reaction was only 30 minutes. It was efficient to produce to 7-amino-3-benzoylindolizine-1, 2-dicarboxylate derivatives. Newly synthesized compounds have been purified by column chromatography and recrystallized from ethyl acetate. The structures have been confermed by elemental analysis and spectroscopic techniques like ${ }^{1} \mathrm{H}-\mathrm{NMR},{ }^{13} \mathrm{C}-\mathrm{NMR}$, LC-MS.

\section{Pharmacological studies}

All synthesized compounds have been tested for antibacterial activity. Among 10 compounds only five compounds

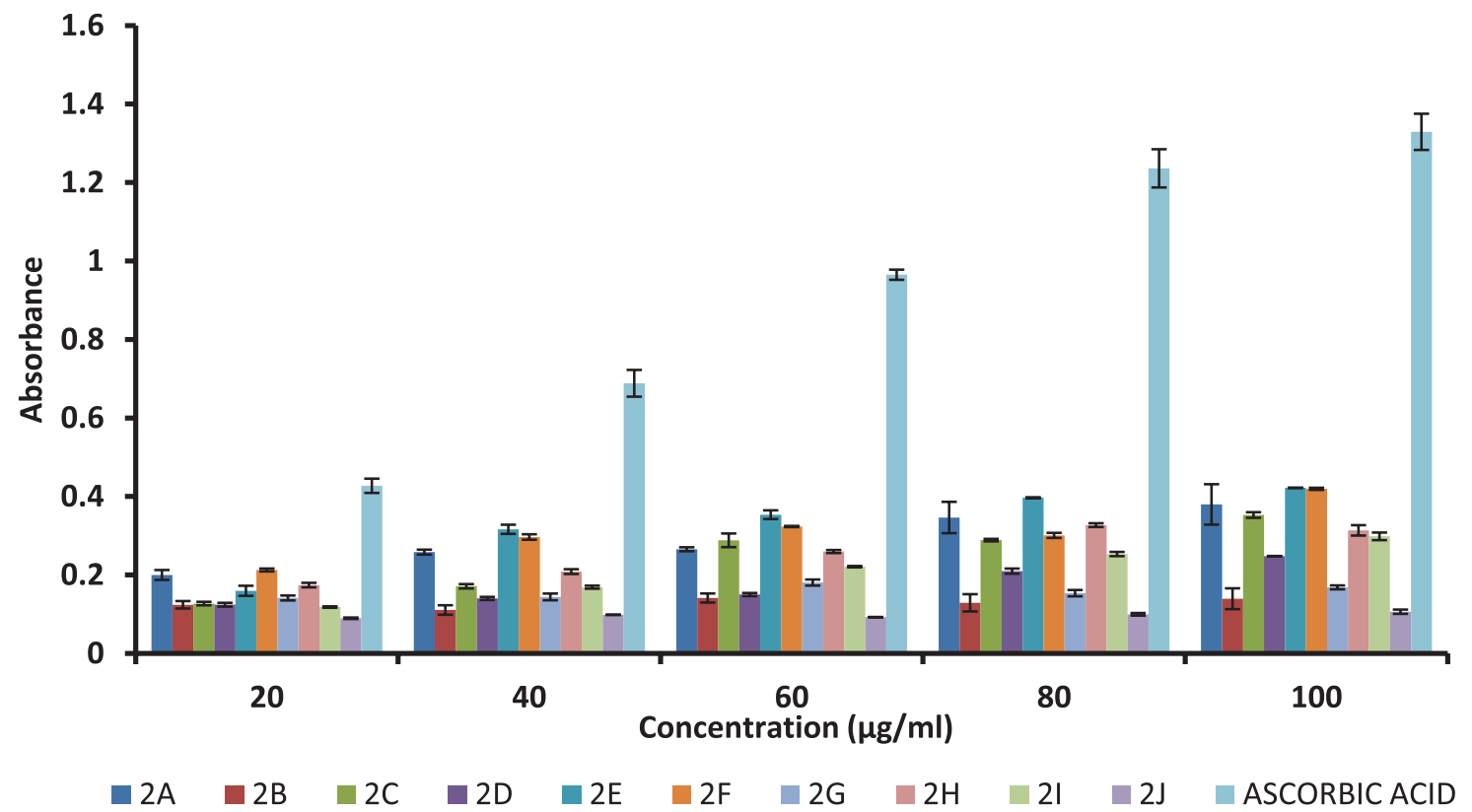

Figure 3. The antioxidant activity of dimethyl 7-amino-3-benzoylindolizine-1,2-dicarboxylate derivatives by reducing power. Values were means \pm SD of triplicate.

Table 6. Lipid peroxidation inhibition activity of synthesized dimethyl 7-amino-3-benzoylindolizine-1,2-dicarboxylate derivatives.

\begin{tabular}{cccccc}
\hline Compd. number & \multicolumn{5}{c}{ Inhibition in \% } \\
\hline Concentration $(\boldsymbol{\mu g} / \mathbf{m l})$ & $\mathbf{2 0}$ & $\mathbf{4 0}$ & $\mathbf{6 0}$ & $\mathbf{8 0}$ & $\mathbf{1 0 0}$ \\
\hline $2 \mathrm{a}$ & 10.71111 & 13.85815 & 15.34055 & 17.12565 & 18.85059 \\
$2 \mathrm{~b}$ & 11.11117 & 14.08192 & 17.25151 & 19.25151 & 22.25181 \\
$2 \mathrm{c}$ & 13.14982 & 14.03798 & 18.95572 & 24.01957 & 24.73153 \\
$2 \mathrm{~d}$ & 10.39262 & 27.38557 & 32.74164 & 34.40364 & 35.06474 \\
$2 \mathrm{e}$ & 18.85522 & 25.62413 & 27.83602 & 33.63016 & 37.10736 \\
$2 \mathrm{f}$ & 10.45268 & 16.12327 & 18.25914 & 29.14443 & 39.39944 \\
$2 \mathrm{~g}$ & 5.362016 & 8.362016 & 13.85958 & 27.67104 & 42.07086 \\
$2 \mathrm{~h}$ & 6.797542 & 25.13023 & 26.84195 & 27.36313 & 28.33041 \\
$2 \mathrm{i}$ & 16.55333 & 23.01244 & 26.77826 & 27.71957 & 29.86410 \\
$2 \mathrm{j}$ & 13.26352 & 19.77354 & 25.78557 & 26.49128 & 29.93738 \\
Ascorbic acid & 31.65604 & 46.5659 & 62.92903 & 71.20411 & 77.75096 \\
\hline
\end{tabular}




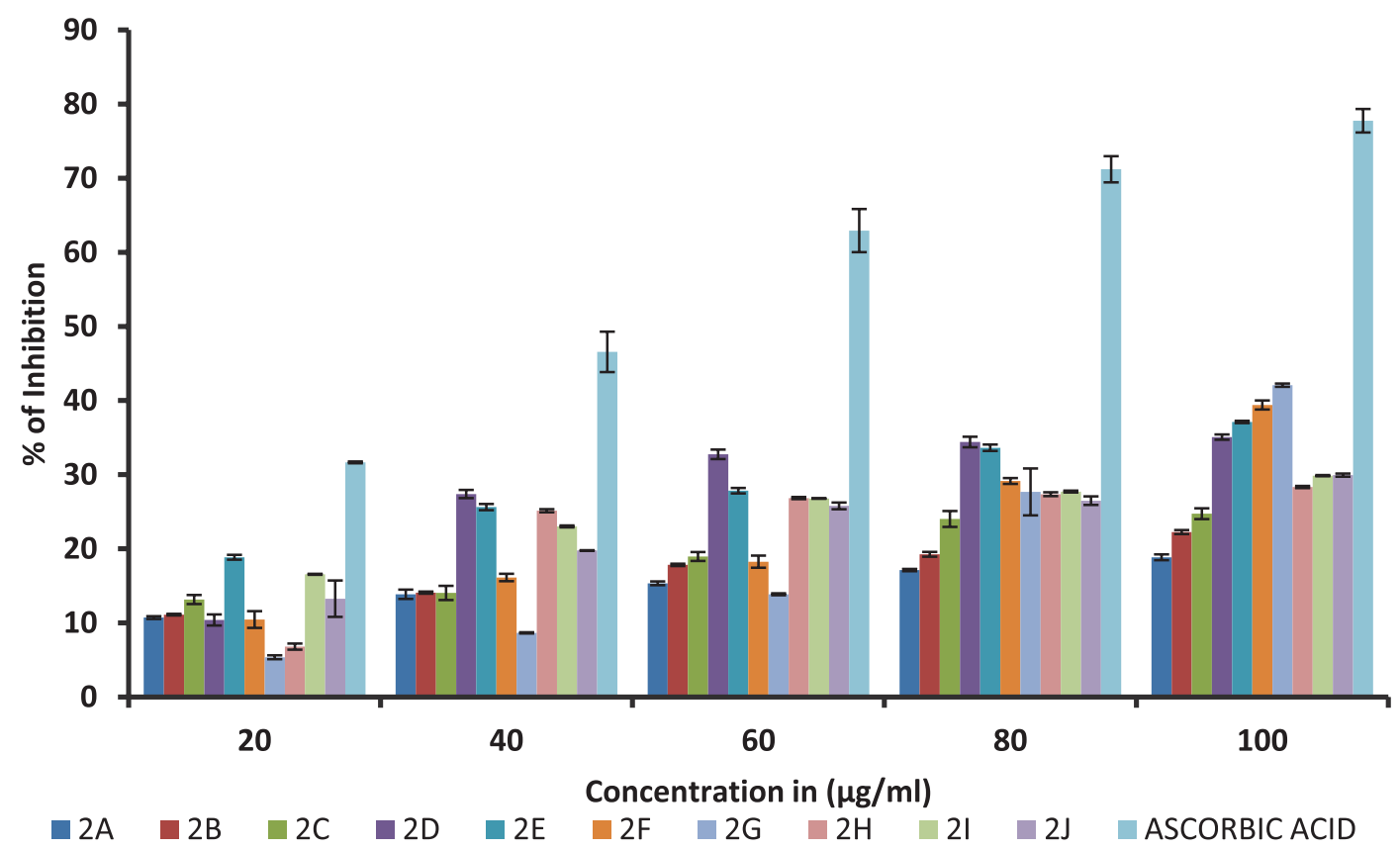

Figure 4. The lipid peroxidation inhibition activity of dimethyl 7-amino-3-benzoylindolizine-1,2-dicarboxylate derivatives. Values were means \pm SD of triplicate.

shows inhibition activity and the other five compounds were non-toxic to bacteria. Compounds $2 \mathrm{e}, 2 \mathrm{~g}$, and $2 \mathrm{i}$ show the highest inhibition zone and compound $2 \mathrm{a}$ and $2 \mathrm{f}$ shows moderate inhibition zone. In Table 2, compounds inhibition zone against bacteria mentioned in $\mathrm{mm}$ to respective bacteria.

The synthesized compounds were screened for their antioxidant activity against DPPH free radical scavenging activity, Nitric oxide free radical scavenging activity, Reducing power scavenging activity and Lipid peroxidation inhibition activity. The 7-amino-3-benzoylindolizine-1,2-dicarboxylate derivatives antioxidant property have been studied by above mentioned four methods. The all the synthesized indolizine derivatives show moderate antioxidant activity in all the methods.

\section{CONCLUSION}

The present research work is concentrated on the efficient synthesis of indolizine derivatives. The eco-friendly reaction method was adapted to synthesized indolizine derivatives. All synthesized 7-amino-3-benzoylindolizine-1,2-dicarboxylate compounds have been tested for their anti-bacterial activity and five compounds possess remarkable activity against selected bacteria Bacillus subtilis, Eschericha coli, Psseudomonas aeruginosa, Staphylococcous aureus, Baccillus cereus and Zymomonas mobilis. The compound $2 \mathrm{f}$ only shows inhibition activity against Bacillus subtilis. The compound $2 \mathrm{a}$ does not shows inhibition activity against Bacilluscereus and it actively inhibits the all other reaming five bacteria. The compound $2 \mathrm{e}, 2 \mathrm{~g}$ and $2 \mathrm{j}$ possess very remarkable activity against all selected bacteria with significant inhibition zone.

All synthesized 7-amino-3-benzoylindolizine-1, 2-dicarboxylate compounds have been tested for their antioxidant activity. All compounds significantly possess antioxidant property in DPPH free radical scavenging activity, Nitric oxide free radical scavenging activity, Reducing power scavenging activity, and Lipid peroxidation inhibition activity. In all the methods, synthesized compounds shows their activity less than standard. It means synthesized 10 compounds shows not negligible antioxidant property in each method.

\section{ACKNOWLEDGMENT}

The authors would like to thank the Rani Channamma University, Belagavi, for providing laboratory facilities. The authors also acknowledge JSS Academy of Higher Education and Research, Mysore for carrying out anti-bacterial activities.

\section{CONFLICT OF INTERESTS}

The authors declared that they have no conflict of interest.

\section{REFERENCES}

Flitsch. W. in Comprehensive Heterocyclic Chemistry. In: Katritzky AR, Rees CW (eds.). Pergamon Press, Oxford, UK, Vol. 4, p 443, 1984.

Hazra A, Mondal S, Maity A, Naskar S, Saha P, Paira R, Sahu KB Paira P, Ghosh S, Sinha C, Samanta A, Banerjee S, Mondal NB. AmberliteIRA-402 $(\mathrm{OH})$ ion exchange resin mediated synthesis of indolizines, pyrrolo [1,2-a] quinolines and isoquinolines: antibacterial and antifungal evaluation of the products. Eur J Med Chem, 2011, 46(6):2132-40.

Kemnitzer W, Kuemmerle J, Jiang S, Zhang HZ, Sirisoma N, Kasibhatla S, Crogan-Grundy C, Tseng B, Drewe J, Cai SX.. Discovery of 1-benzoyl-3-cyanopyrrolo[1,2-a]quinolines as a new series of apoptosis inducers using a cell- and caspase-based high-throughput screening assay. Part 1: Structure-activity relationships of the 1- and 3-positions. Med Chem Lett, 2008; 18:6259.

Koleva II, van Beek TA, Linssen JP, de Groot A, Evstatieva LN. Screening of plant extracts for antioxidant activity: a comparative study on three testing methods. Phytochem Anal, 2002; 13(1):8-17. 
Mallikarjuna SM, Sandeep C, Basavaraj P. Synthesis, antimicrobial activity of piperazin-1-yl (3,4,5-trimethoxyphenyl)methanone derivatives. Der Pharma Chemica, 2016; 8:262-8.

Marcocci L, Maguire JJ, Droy-Lefaix MT, Packer L. The nitric oxide scavenging properties of Ginkgo biloba extract EGb761. Biochem. Biophys. Res Commun, 1994; 201:748-55.

Nagesh HK, Basavaraj P, Sandeep C, Musturappa TE, Lokesh MR. Synthesis and characterization of novel benzothiophene substituted oxadiazole derivatives and their antimicrobial activity. Der Pharma Chemica, 2015; 7:129-36.

Nagesh HK, Basavaraj P, Sandeep C, Yuvaraj TCM, Siddesh MB, Mallikarjuna SM. Synthesis and antimicrobial activity of benzothiophene substituted coumarins, pyrimidines and pyrazole as new scaffold. Int J Pharm Sci Rev Res, 2014; 28:6-10.

Oyaizu M. Studies on products of browning reactions: antioxidative activities of products of browning reaction prepared from glucosamine. Jpn J Nutr, 1986; 44(6):307-15.

Ruberto G, Baratta MT, Deans SG, Dorman HJ. Antioxidant and antimicrobial activity of Foeniculum vulgare and Crithmum maritimum essential oils. Planta Med 2000; 66(8):687-93.

Rakshita BK, Pruthvira RD, Swamy MT, Uppar V, Chandrashekharappa S. Corrosion inhibition studies of Al 356 alloy by Using novel synthesize d n2-phenyl-1,3,5-triazine- 2,4-diamine. Int J Res Anal Rev, 2019; 6:784-92.

Sandeep C, Padmashali B, Kulkarni RS. Synthesis of isomeric subtituted 6-acetyl-3-benzoylindolizine-1-carboxylate and 8-acetyl-3benzoylindolizine-1-carboxylate from subtituteded 3-acetyl pyridinium bromides and their antimicrobial activity. J Appl Chem, (Lumami, India), 2013a; 2:1049-56.

Sandeep C, Basavaraj P, Rashmi SK, Mallikarjuna SM, Siddesh MB, Nagesh HK, Thriveni, KS. Synthesis of substituted 5-acetyl-3benzoylindolizine-1-carboxylate from substituted 2-acetyl pyridinium bromides. Heterocycl Lett, 2014; 4:371-6.

Sandeep C, Padmashali B, Kulkarni RS. Efficient synthesis of indolizines and new imidazo[1,2-a]pyridines via the expected cyclization of aromatic cycloimmonium ylides with electron deficient alkynes and ethyl cyanoformate. Tetrahedron Lett, 2013b; 54:6411-4.

Sandeep C, Venugopala KN, Tratrat C, Mahomoodally FM, Aldhubiab BE, Haroun M, Venugopala R, Mohan MK, Kulkarni RS, Attimarad MV, Harsha S, Odhav B. Efficient synthesis and characterization of novel indolizines: exploration of in vitro COX-2 inhibitory activity and molecular modelling studies. New J Chem, 2018a; 42:4893-901.

Sandeep C, Venugopala KN, Nayak SK, Gleiser RM, García DA, Kumalo HM, Kulkarni, RS, Mahomoodally FM, Venugopala R, Mohan MK, Odhav B. One-pot microwave assisted synthesis and structural elucidation of novel ethyl 3-substituted-7-methylindolizine-1-carboxylates with larvicidal activity against Anopheles arabiensis. J Mol Struct, 2018b; 1156:377-84.
Sandeep C, Venugopala KN, Gleiser RM, Chetram A, Padmashali B, Kulkarni RS, Venugopala R, Odhav B. Greener synthesis of indolizine analogues using water as a base and solvent: study for larvicidal activity against Anopheles arabiensis. Chem Biol Drug Des, 2016b; 88:899-904.

Sandeep C, Padmashali B, Venugopala KN, Kulkarni RS, Venugopala R, Odhav B. Synthesis and characterization of ethyl 7-acetyl2-substituted 3-(substituted benzoyl)indolizine-1-carboxylates for in vitro anticancer activity. Asian J Chem, 2016a; 28:1043-48.

Siddesh MB, Basavaraj P, Thriveni KS, Sandeep C. Synthesis of polynuclear pyrimidine derivatives and their pharmacological activities. Heterocycl Lett, 2014a; 4:503-14.

Siddesh MB, Basavaraj P, Thriveni KS, Sandeep C, Goudarshivnnanava BC. Synthesis and pharmacological evaluation of some novel pyrimidine derivatives. J Applicable Chem, 2013; 2:1281-8.

Siddesh MB, Padmashali B, ThrivenI KS, Sandeep C. Synthesis of thiophene-linked pyrimidopyrimidines as pharmaceutical leads. J Chem Sci, 2014b; 126:821-6.

Swinborne FJ, Hunt JH, Klinkert G. Adv Heterocycl Chem, $1978 ; 23: 103-70$

Thriveni KS, Padmashali B, Siddesh MB, Sandeep C. Synthesis of pyrimidine incorporated piperazine derivatives and their antimicrobia activity. Indian J Pharm Sci, 2014; 76:332-8.

Venugopala KN, Chandrashekharappa S, Pillay M, Bhandary S, Kandeel M. Mahomoodally FM, Morsy MA, Chopra D, Bandar EA, Attimarad M, Alwassil OI, Harsha S, Mlisana K, Odhav B. Synthesis and structural elucidation of novel benzothiazole derivatives as anti-tubercular agents: In-silico screening for possible target identification. Med Chem, 2019; 15(3): 311-26

How to cite this article:

Uppar V, Chandrashekharappa S, Basarikatti AI, Banuprakash G, Mohan MK, Chougala M, MudnakuduNagaraju KK, Ningegowda R, Padmashali B. Synthesis, antibacterial and antioxidant studies of 7-amino-3-(4fluorobenzoyl)indolizine-1-carboxylate derivatives. J Appl Pharm Sci, 2020, 10(02):077-085. 